\title{
3 Research Soure \\ Identification of Potential Biomarkers and Pathways in Neonatal Hypoxic-Ischemic Brain Injury: Based on Bioinformatics Technology
}

\section{Shangbin Li}

The First Hospital of Hebei Medical University

Shuangshuang Li

Chengdu University of Traditional Chinese Medicine

Qian Zhao

The First Hospital of Hebei Medical University

Jiayu Huang

The First Hospital of Hebei Medical University

Jinfeng Meng

The First Hospital of Hebei Medical University

Weichen Yan

The First Hospital of Hebei Medical University

Jie Wang

The First Hospital of Hebei Medical University

Changjun Ren ( $\nabla$ 137544907@qq.comChangjun )

The First Hospital of Hebei Medical University

Ling HaO

The First Hospital of Hebei Medical University

\section{Research Article}

Keywords: neonatal hypoxic-ischemic brain injury, inflammatory response, immune response, differentially expressed genes, bioinformatics analysis

Posted Date: August 5th, 2021

DOI: https://doi.org/10.21203/rs.3.rs-777916/v1

License: (c) (1) This work is licensed under a Creative Commons Attribution 4.0 International License.

Read Full License 


\section{Abstract}

\section{Background}

Neonatal hypoxic-ischemic brain damage (HIBD) is one of the most common serious diseases in newborns, with a high mortality and disability rate. This study aims to use the bioinformatics analysis to identify potential hematologic/immune systems tissue-specific genes and related signaling pathways neonatal HIBD.

\section{Methods}

Microarray datasets in HIBD were downloaded from the Gene Expression Omnibus database, and DEGs were identified by $\mathrm{R}$ software.Enrichment analyses were performed and protein-protein interaction networks were constructed to understand the functions and enriched pathways of DEGs and to identify central genes and key modules.

\section{Results}

In the cerebral cortex tissue with HIBD, 2598 DEGs were identified, including 2362 up-regulated and 236 down-regulated DEGs. In the blood with HIBD, 1442 DEGs were identified, including 540 up-regulated and 902 down-regulated DEGs. The results of biological processes and KEGG enrichment were very similar in DEGs of the two kinds of tissues, and both involved inflammation, immunity and apoptosis. The common DEGs of the two kinds of tissues also showed similar results in biological processes and KEGG enrichment.and four hematologic/immune system tissues specifically expressed potential biomarker genes were confirmed through a variety of methods, which were verified by GEO datasets and published experimental research.

\section{Conclusion}

The DEGs of HIBD including the potential peripheral biomarkers TYROBP, ITGAM, EGR1 and HMOX1, which may play a role in the pathogenesis of HIBD through inflammation and immune-mediated signaling pathways.

\section{Introduction}

Hypoxic ischemic brain injury (HIBD), also known as hypoxic-ischemic encephalopathy (HIE), is one of the most common serious diseases in newborns. In developed countries, HIBD occurs in 1-8 cases per 1,000 live births, and 10-26 cases per 1,000 in low and middle income countries[1-2]. In the long term, HIBD can cause cerebral palsy, epilepsy, visual impairment and motor cognitive impairment[3]. At present, neonatal HIBD is mainly based on the clinical manifestations of the nervous system, imaging and electrophysiological examinations to diagnose and predict prognosis[4]. These methods of diagnosis and prognostic judgment require a long time and cause delay in diagnosis. So that delay in diagnosis oftentimes leads to that many HIBD babies miss the optimal treatment time, which may cause different 
levels of neurological sequelae.With the rapid development of biomedicine, there have been many reports on biomarkers related to neonatal HIBD[5]. However, there is no blood-based marker that is stable enough to diagnose HIBD or predict prognosis[6]. Currently, there is still a lack of effective drugs to treat HIBD, and prevention is the key to this disease. In recent years, mild hypothermia neuroprotective therapy has become the standard for clinical treatment of HIBD, and other adjuvant therapies such as erythropoietin and neuroprotective agents are rapidly transitioning from basic science to clinical application[7-8]. However, pharmacological treatments were solely focused on neurons. Gradually, glial cells started to be considered as alternative targets for neuroprotection[9-10].

More and more evidences show that hypoxia-ischemia can induce inflammation in the brain parenchyma and peripheral immune system, which plays a vital role in mediating secondary neuronal death[1112].The brain is considered a place of immune privilege because the blood-brain barrier restricts bloodborne cells and proteins from entering the central nervous system (CNS)[13].In the central nervous system, microglia, astrocytes, and oligodendrocytes play the roles of inflammatory cells and immune cells. There are currently studies that the sequelae of the nervous system are related to the activation of astrocytes[14].The inflammatory response of the brain can lead to the activation of astrocytes and the activation and aggregation of microglia to provide immune defense for nerve cells, which can lead to rapid changes in their gene expression[15].The research team of Dr. Ting Qi and Professor Jian Yang found that the blood has genetic targets that can be used to identify brain-related properties (such as IQ) and diseases (such as schizophrenia)[16].This discovery provides a new research idea for brain disease research.

Therefore, this study aims to apply the public gene expression comprehensive database (GEO) for bioinformatics analysis to determine the differentially expressed genes (DEGs) in neonatal cerebral cortex tissue and blood.Our research study focused on finding the potential hematologic/immune system tissue specificity gene and related signal pathways that are closely related to HIBD and revealing the underlying molecular mechanisms.

\section{Materials And Methods}

\subsection{Microarray Data}

The expression profile datasets of HIBD were searched from the publicly available Gene Expression Omnibus (GEO) database (http://www.ncbi.nlm.nih.gov/geo). The microarray datasets generated from the cerebral cortical in rats with HIBD and from the whole blood in human with HIBD and controls were used in the study. Four gene expression profiles include GSE37777, GSE18356, GSE121178 and GSE112137 were obtained.The details of the datasets are shown in Table 1.

\subsection{Identification of differentially expressed genes[DEGs $\square$}

We analyzed the DEGs of healthy and HIBD samples in the cerebral cortex tissue and blood by using the limma software package, especially the raw data of datasets GSE121178 and GSE37777 were extracted 
with the affy package and individually normalized with the robust multi-array average (RMA) package, and batch effects were eliminated between experiments by applying the ComBat function in the SVA package. The DEGs cut-off thresholds of both are set as follows: (1) $p<0.05$; (2) $\log _{2}$ (FoldChange, FC) >

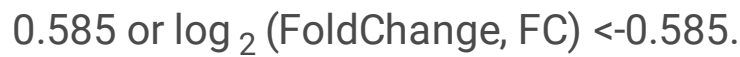

\subsection{GO Enrichment and KEGG Pathway Analysis}

Gene ontology (GO) includes three types of data, namely, cellular components (CC), molecular functions (MF), and biological processes (BP)[17]. GO is often used to annotate genes according to a defined set of structured words. The Kyoto Encyclopedia of Genes and Genomes (KEGG) is used to match the information of gene pathways[18]. We used the DAVID 6.8 (https://david.ncifcrf.gov) online tool[19] to perform GO biological process (GO-BP) analysis on DEGs in HIBD, and use KOBAS 3.0 (http://kobas.cbi.pku.edu.cn/) online tool for KEGG pathway analysis[20]. $\mathrm{P}<0.05$ was selected as the threshold to identify the significant enrichment of GEGs in GO terms and signal pathways.

\subsection{PPI network construction, central genes and key module Analysis}

The tool for searching gene/protein interactions (STRING: http://string-db.org/) is an online biological database designed to evaluate the protein-protein interaction(PPI) network. Intersecting the DEGs of cerebral cortex tissue and whole blood to obtain a common DEGs. According to the information in the STRING database (PPI score> 0.4), we selected the protein related to the common DEGs (species: Homo), and used the Cytoscape software (http://cytoscape.org/ ) build a PPI network. Then we used five methods(degree, Maximum Neighborhood Component (MNC), Radiality centrality, Stress centrality, Closeness centrality) to sequence and evaluate central genes . In addition, we used the MCODE algorithm to obtain key modules.

\subsection{Validation of central genes and determination of tissue-specific biomarker genes}

We identified the central genes by using the Genecards database[21] and further determine the genes potentially related to the disease. The central genes was located for further determine the central genes expressed in the hematologic/immune system by using the Biogps database[22].The results obtained from the two databases were integrated to obtain HIBD hematologic/immune system tissue specific biomarker genes. We applied the GSE121137 data set for external verification of tissue-specific genes.Box-plot analysis and receiver operating characteristic(ROC) curve were performed to display tissue-specific genes for external verification of the diagnostic potential of HIBD by using GraphPad Prism8.0 soft.

\subsection{Prediction of transcription factors and miRNAs of key central genes}

The CHEA3 database[23] are used to predict the relationship between key central genes and transcription factors (TF); The mirDIP database[24] was identified to predict the relationship between key central genes 
and miRNA.Network analysis was performed by running Cytoscape application,and the key TF and key miRNA was determined according to the Degree value.

\section{Results}

\subsection{Identification of Differentially Expressed Genes (DEGs)}

In the cerebral cortex tissue, 2598 DEGs were screened, including 2362 up-regulated DEGs and 236 downregulated DEGs. In the blood, 1442 DEGs were screened, including 540 up-regulated DEGs and 902 downregulated DEGs. The volcano map in Figure 1A-B displays the general distribution of these genes, and expression of the top 30 up-regulation DEGs and the top 30 down-regulation DEGs of two kinds of samples were showed in the heatmap in Figure 1C-D.

\subsection{GO Term Enrichment Analysis and KEGG Pathway Analysis}

We applied the DAVID online tool and the KOBAS online tool to cluster the GO protein function and the KEGG signal pathway of the DEGs of HIBD. As shown in GO analysis results (Figure 2A-B,only the top 30 items of gene enrichment were shown), DEGs in cerebral cortex tissue cell samples were mainly enriched in biological processes such as cell signal transduction, transcription regulation, redox response, inflammatory response, immune response, cell proliferation, cell apoptosis, drug response and multicellular biological development; DEGs in blood samples were mainly enriched in biological processes such as redox reaction, inflammation, angiogenesis, multicellular biological development, cell morphology regulation, transmembrane transport, positive regulation of the MAPK cascade, and positive regulation of the Notch signaling pathway. KEGG enrichment results showed(Figure 2C-D, only showing the top 30 items of gene enrichment): DEGs in cerebral cortex tissue cell samples were mainly in metabolic signaling pathways, cancer pathways, cytokine-cytokine receptor interactions, PI3K-Akt signaling pathway,neural active ligand-receptor interaction pathway, MAPK signaling pathway,Rap1 signaling pathway,chemokine signaling pathway, and infection-related signaling pathways; DEGs in blood samples were mainly enriched in metabolic signaling pathways, cancer pathways, neuroactive ligand-receptor interaction pathways, PI3K-Akt signaling pathways, endocytosis, Rap signaling pathways, calcium signaling pathways, and infection-related signaling pathways. Other results are in the supplementary materials

In general, the enrichment results of GO and KEGG of DEGs in the cerebral cortex tissue and DEGs in whole blood are related to hypoxic-ischemic brain injury, and both involve inflammation, immunity and apoptosis.

\subsection{Common DEGs identified in the cerebral cortex tissue and blood of HIBD cases}

There were 86 genes overlapping between DEGs in the cerebral cortex tissue with HIBD and DEGs in whole blood with HIBD. As shown in Figure 3, these 86 common DEGs included 67 up-regulation and 19 down-regulation.The biological processes that these common DEGs mainly involved inflammation and 
immunity (Figure 4A, only showing the top 15 items in terms of gene enrichment), including participation in neutrophils, macrophage-mediated inflammation, platelet activation, inflammatory cells activation,participates in immune response, regulation of lymphocyte differentiation. Cross-examination of common DEGs and GO biological process terms showed (Figure 4B) that some genes related to inflammation were also enriched in immune-related biological processes, indicating that these genes may be related to the inflammation-immune damage mechanism in HIBD.The enrichment results of cellular components and molecular functions (Figure 4C) showed that the cellular components of common DEGs were mainly enriched in plasma membrane, extracellular body, cytoskeleton, extracellular zone, cell surface, extracellular space, and oscillating cilia; molecular functions mainly included binding to phosphotyrosine, phosphatase, interleukin-1, serine endopeptidase activity, phospholipase D activity, interleukin-1 receptor activity. Similarly, common DEGs were significantly enriched in the KEGG pathway (Figure 4D, showing the signal pathway with FDR<0.05), mainly involving microbial infection, natural killer cell-mediated cytotoxicity, osteoclast differentiation, cancer pathway, FceRI signaling pathways, complement and coagulation cascade pathways, hematopoietic cell spectrum, neuroactive ligand receptor interaction, Rap1 signaling pathway.

\subsection{Construction of protein-protein interaction (PPI) network, identification of central genes and key modules}

PPI network of 86 common DEGs was constructed(Figure 5A), including 50 nodes and 89 edges. Network topology analysis was applied to arrange the nodes according to the degree of connection. The larger the Degree, the larger the dot.Then, according to the 5 centrality methods (Figure 5B), We determined 10 common genes (ITGAM, SYK, PLD1, KLRD1, NCKAP1L, EGR1, MAPK3, TYROBP, HMOX1, IL1R1) among the first 20 nodes as central genes. Then we performed subnet module analysis (filter criteria: Degree Cutoff=3, node score=0.2, K-core=2, Max Depth=100). Two key modules were obtained (Cluster1 $=3.70$, Cluster2 $=2.80$ ) (Figure $5 \mathrm{C}-\mathrm{D}$ ), and they contained $8 \mathrm{DEGs}$, which contained 4 central genes (red).DAVID and KOBAS databases were used for the enrichment analysis of GO and KEGG pathways for 8 DEGs in these two modules. The results showed (Table 2.): The GO-BP of these two modules GO mainly involved integrin-mediated signaling pathways, positive regulation of chemokine biosynthesis, and response to hypoxia;The results of KEGG mainly involved HIF-1 signaling pathway, hepatocellular carcinoma, Rap1 signaling pathway, neomycin, kanamycin and gentamicin biosynthesis, galactose metabolism, etc.

\subsection{Validation of key central genes and determination of hematologic/immune system tissue-specific biomarker genes}

Using Genecards database to identify 10 central genes, it was found that 7 central genes may be related to HIBD, and no related reports were found for the other 3 genes. Intersecting these 7 genes with the genes of 2 modules to get 4 genes. It was confirmed in the Biogps database that all 4 genes were clearly expressed in the hematologic/immune system, and these genes were defined as HIBD hematologic/immune system tissue specificity potential biomarkers (Table 3 ).In this study, 32 samples 
in the GSE12137 data set (hypoxia model of early cortical progenitor cells) were used to verify 4 potential biomarkers, and it was found that two potential biomarkers were significantly up-regulated. The box plots of the two genes (Figure 6A-B) were clearly differentially expressed in the hypoxia model of cortical progenitors cells; the ROC curve (Figure 6C-D) showed that the two genes have good performance in identifying cases and controls $₫ A \cup C_{E G R 1}=0.8398, A U_{\mathrm{HMOX}_{1}}=0.9063 \bigotimes$.In addition, a literature search was conducted on ITGAM and TYROBP that have not been verified by the data set, and related studies (qt-PCR test) showed that ITGAM and TYROBP were significantly expressed in the brain tissue of the hypoxicischemic brain injury model[25].This study believed that these 4 genes may be specific potential biomarker genes for the diagnosis of hematologic/immune system in HIBD.Especially, EGR1 and HMOX1 may have a diagnostic effect on early HIBD

\subsection{Prediction of transcription factors and miRNAs of key central genes}

According to the comprehensive ranking of multiple sub-databases in the CHEPA3 database, the top 10 potential transcription factors with higher scores are selected to construct an interaction network (Figure $7 A)$.In the mirDIP database, we set the credibility parameter to very high, and obtained at least 88 miRNAs predicted by 7 sub-databases (Figure 7B). 9 key transcription factors (TFEC, SPI1, CEBPE, HLX, ZNF438, ZNF641, CEBPB, ELF4, LYL1) and 3 key miRNAs (hsa-miR-218-5p, hsa-miR-217, hsa-miR-377-3p) were screened according to the topoisomeric method,and their interactive network may be a potential key regulatory pathway in the pathogenesis of HIBD.

\section{Discussion}

Neonatal hypoxic-ischemic brain injury is an important cause of neonatal death and disability[26]. The pathogenesis of HIBD is very complicated. At present, there are no stable diagnostic biomarkers and specialized treatment methods for neonatal HIBD. So far, many studies have reported that inflammatory response and immune response play a key role in the development and recovery of hypoxic-ischemic brain injury. The activation of immune cells and the release of cytokines in the central and peripheral immune systems were involved[27-28].

This research obtained mRNA expression data about HIBD from the Public Gene Expression Comprehensive (GEO) database for bioinformatics analysis,and DEGs were identified in normal and case from cerebral cortex tissue samples and blood samples. We performed GO function annotation and KEGG pathway enrichment analysis on the DEGs of the cerebral cortex tissue and blood respectively.Regardless of GO function annotation or KEGG analysis, the functions and signal pathways related to inflammation, immune response and apoptosis were screened in the DEGs of the cerebral cortex tissue and blood, in particular, PI3K-Akt signaling pathway, MAPK signaling pathway, Rap signaling pathway and other classic signaling pathways have been reported to be related to brain injury[2930].This similarity provided a feasible basis for exploring genetic changes that reflect cerebral hypoxicischemic damage in the blood. 86 overlapping genes were obtained in their DEGs. The GO biological process of common DEGs also involved inflammation and immune response, such as participation in 
neutrophil, macrophage-mediated inflammation, platelet activation, inflammatory cell activation, immune response lymphocyte differentiation regulation,etc;The KEGG signaling pathways mainly included natural killer cell-mediated cytotoxicity, osteoclast differentiation, FcعRI signaling pathway, complement and coagulation cascade pathways. We believed that the path most related to the disease is the natural killer cell-mediated cytotoxicity pathway. It was found in the KEGG mapper database that its downstream can trigger the apoptosis pathway[31].Unfortunately, the correlation between the natural killer cell-mediated cytotoxicity pathway and HIBD had not been confirmed by relevant experimental studies, and further studies on animal experiments or clinical experiments are still needed.

The PPI network of Common DEGs was constructed through the String database and visualized through cytoscape. Ten central genes (ITGAM, SYK, PLD1, KLRD1, NCKAP1L, EGR1, MAPK3, TYROBP, HMOX1, IL1R1) were identified through five sequencing methods in cytocentralba.In addition, we analyzed the PPI network of the common DEG and identified 2 key modules through MCODE (score $\geq 3$ ). Then use DAVID and KOBAS to analyze the GO and KEGG pathway enrichment analysis of DEGs in the two modules.Our results show that these two modules are involved in biological processes such as integrin-mediated signaling pathways, positive regulation of chemokine biosynthesis processes, and responses to hypoxia.Integrin is a type I heterodimeric transmembrane protein composed of $a$ and $\beta$ subunits[32].Previous studies have shown that the regulation of integrin-mediated signaling pathways such as MAPK and PI3K signaling pathways can resist mitochondrial apoptosis in hypoxic cardiomyocytes[33].Chemokines are a family of small proteins (8-10 kDa).It has been discovered earlier that the expression of some chemokines such as macrophage inflammatory protein-1 precedes the infiltration of immune cells into the brain and participates in the inflammatory response[34].KEGG results mainly involved HIF-1 signaling pathway, hepatocellular carcinoma, Rap1 signaling pathway, neomycin, kanamycin and gentamicin biosynthesis, galactose metabolism and other signaling pathways.The HIF-1 signaling pathway is involved in the transcriptional regulation of many genes after hypoxia/ischemia, and it also plays many different roles in protecting the brain during hypoxia/ischemia preconditioning, including vascular regulation, apoptosis regulation, oxidation, excitement,sexual toxicity regulation, immune regulation and so on[35].

Combining the 10 Central genes with the genes of the two key modules, we identified 4 key central genes TYROBP, ITGAM, ERG1 and HMOX1.At the same time, the Biogps database was used to test their expression levels in the hematologic/immune system.TYROBP, ITGAM, ERG1 and HMOX1 are central proteins in the PPI network, and are also common DEGs in HIBD cortical tissue and whole blood.We define these genes as HIBD hematologic/immune system tissue specific biomarkers. In this study, TYROBP participated in the natural killer cell-mediated cytotoxicity signaling pathway and hematopoietic cell spectrum signaling pathway.TYROBP is a transmembrane signal transduction adaptor protein, which has both pro-inflammatory and anti-inflammatory effects[36].Albertsson's study found that after $\mathrm{HI}$ injury, high expression of TYROBP was found in the damaged area, including the subcortical white matter area, hippocampus, periventricular area, and meningeal area,and may be involved in the occurrence of white matter damage in premature infants[37].In this study, ITGAM participated in the cell adhesion molecule pathway, the leukocyte transendothelial migration pathway, and the Rap1 signaling pathway.Microglial 
cells increase significantly in mice and human brain injury and neurodegenerative diseases, and ITGAM expression also shows a positive change[38].Previous studies reported that the protein CD11B encoded by ITGAM was significantly increased after $\mathrm{HI}$ injury, and ITGAM has a pro-inflammatory effect in the mechanism of $\mathrm{HI}$ injury[39]. ITGAM can be combined with TYROBP in microglia to control the production of superoxide ions in microglia, thereby promoting neuronal apoptosis[40]. In this study, HMOX1 participated in the HIF-1 signaling pathway and angiogenesis pathway. HMOX1 has anti-inflammatory, anti-oxidative stress and anti-proliferation effects, can promote the survival of neurons and oligodendrocytes, and can reduce the degree of HIBD nerve damage[41-42]. In this study, EGR1 participated in the interleukin-1 mediated signaling pathway and $\mathrm{GnRH}$ signaling pathway.Previous studies have shown that ERG1 induced by hypoxia-ischemia can reduce the expression of BDNF and aggravate brain damage[43]. We applied the GEO data set to verify 4 hematologic/immune systemspecific genes and indirectly confirmed the continuous differential expression of ERG1 and HMOX1 in the pathogenesis of HIBD (24h-8 weeks). ROC curve analysis shows that ERG1 and HMOX1 have high diagnostic value for early HIBD.However, TYROBP and ITGAM have not been validated in the data set, which may have a certain relationship with the number of in vitro cell models in the data set deviating from the hematologic/immune system regulation. Published experimental documents have confirmed that they are significantly highly expressed in the $\mathrm{HI}$ model.In general, these 4 potential blood/immune biomarker genes as candidate molecular targets may provide potential references for the diagnosis, treatment and prognosis of BIBD. ERG1 and HMOX1 may be the potential hematology/immune system specific expression of early HIBD. However, further large-scale experiments are needed to verify and clarify their specific mechanisms.

This study also constructed a mutual regulatory network of key central DEGs-TFs and key central DEGsmiRNAs to identify their transcriptional and post-transcriptional regulatory factors.And identified their key transcription factors (TFEC, SPI1, CEBPE, HLX, ZNF438, ZNF641, CEBPB, ELF4, LYL1) and key miRNAs (hsa-miR-218-5p, hsa-miR-217, hsa-miR-377 -3p). miRNAs are non-coding RNA molecules that play a vital role in regulating a series of basic cellular processes, which may induce RNA silencing or act as regulators after DNA transcription[44]. Looney's study confirmed that the expression profile of miRNA in the cord blood of newborn HIE can be changed[45]. Currently, studies showed that hsa-miR-217 can target EGR1 to reduce the inflammatory damage of endothelial cells induced by oxidized LDL[46]. Moreover, it is shown that Overexpression of hsa-miR-217 aggravates hypoxia-induced cardiomyocyte damage by inhibiting the expression of Silent Information Regulator 1[47]. However, no relevant reports have been found for these key miRNAs in hypoxia-induced brain damage. In the regulatory network, the synergistic effect of TF and miRNA is largely unclear.The identification and analysis of miRNA and TF may also reveal the potential pathogenesis of HIBD at the molecular level, and help promote the diagnosis, prognosis and treatment of HIBD.

\section{Conclusion}

In summary, the DEGs of HIBD, including potential peripheral biomarkers TYROBP, ITGAM, EGR1 and HMOX1, may play a role in the pathogenesis of HIBD through inflammation and immune-mediated 
signaling pathways. These potential regulatory genes and models may provide enlightenment for the discovery of new molecular targets for the diagnosis, prognosis and treatment of HIBD, and new breakthroughs in anti-inflammatory and immunotherapy are expected in the future.In order to clarify the role of these new peripheral marker genes in HIBD, more in vitro and in vivo studies and verifications are needed.

\section{Declarations}

\section{Data Availability}

The data used to support the findings of this study are included within the article.

\section{Author statement}

Shangbin Li,Shuangshuang Li and Qian Zhao contributed equally to this work and are co-first authors. Shangbin Li and Qian Zhao searched datasets from the public gene expression comprehensive database.Shangbin Li, Shuangshuang Li and Qian Zhao wrote the manuscript and analyzed the data. Jiayu Huang, Jinfeng Meng, Weichen Yan, and Jie Wang performed the data extraction and the results visualization. Changjun Ren and Ling Hao designed the study and amended the paper. All authors are responsible for reviewing data. All authors read and approved the final manuscript.

\section{Funding}

This study was funded by the government-funded clinical medicine outstanding talent training project leader (team) project in 2020, which was named "Study on the expression and mechanism of microRNA in neonatal bilirubin encephalopathy" [NO.LS202006].

\section{Conflicts of Interest}

The authors declare that there is no conflict of interest.

\section{References}

1. Greco P, Nencini G, Piva I, Scioscia M, Volta CA, Spadaro S, Neri M, Bonaccorsi G, Greco F, Cocco I, Sorrentino F, D'Antonio F, Nappi L. Pathophysiology of hypoxic-ischemic encephalopathy: a review of the past and a view on the future. Acta Neurol Belg. 2020 Apr;120(2):277-288.

2. Kurinczuk JJ, White-Koning M, Badawi N. Epidemiology of neonatal encephalopathy and hypoxicischaemic encephalopathy. Early Hum Dev. 2010 Jun;86(6):329-38.

3. Kurinczuk JJ, White-Koning M, Badawi N. Epidemiology of neonatal encephalopathy and hypoxicischaemic encephalopathy. Early Hum Dev. 2010 Jun;86(6):329-38.

4. Douglas-Escobar M, Weiss MD. Hypoxic-ischemic encephalopathy: a review for the clinician. JAMA Pediatr. 2015 Apr;169(4):397-403. 
5. Lv H, Wang Q, Wu S, Yang L, Ren P, Yang Y, Gao J, Li L. Neonatal hypoxic ischemic encephalopathyrelated biomarkers in serum and cerebrospinal fluid. Clin Chim Acta. 2015 Oct 23;450:282-97.

6. Murray DM. Biomarkers in neonatal hypoxic-ischemic encephalopathy-Review of the literature to date and future directions for research. Handb Clin Neurol. 2019;162:281-293.

7. Douglas-Escobar M, Weiss MD. Hypoxic-ischemic encephalopathy: a review for the clinician. JAMA Pediatr. 2015 Apr;169(4):397-403.

8. Yıldız EP, Ekici B, Tatlı B. Neonatal hypoxic ischemic encephalopathy: an update on disease pathogenesis and treatment. Expert Rev Neurother.2017 May;17(5): 449-459.

9. Herrera MI, Mucci S, Barreto GE, Kolliker-Frers R, Capani F. Neuroprotection in Hypoxic-Ischemic Brain Injury Targeting Glial Cells. Curr Pharm Des. 2017;23(26): 3899-3906.

10. Cho KH, Davidson JO, Dean JM, Bennet L, Gunn AJ. Cooling and immunomodulation for treating hypoxic-ischemic brain injury. Pediatr Int. $2020 \mathrm{Jul} ; 62$ (7):770-778.

11. Hedtjärn M, Mallard C, Hagberg H. Inflammatory gene profiling in the developing mouse brain after hypoxia-ischemia. J Cereb Blood Flow Metab. 2004 Dec;24(12):1333-51.

12. Algra SO, Groeneveld KM, Schadenberg AW, Haas F, Evens FC, Meerding J, Koenderman L, Jansen NJ, Prakken BJ. Cerebral ischemia initiates an immediate innate immune response in neonates during cardiac surgery. J Neuroinflammation. 2013 Feb 7;10:24.

13. Raivich G, Bohatschek M, Kloss CU, Werner A, Jones LL, Kreutzberg GW. Neuroglial activation repertoire in the injured brain: graded response, molecular mechanisms and cues to physiological function. Brain Res Brain Res Rev. 1999 Jul;30(1):77-105.

14. Barateiro A, Chen S, Yueh MF, Fernandes A, Domingues HS, Relvas J, Barbier O, Nguyen N, Tukey RH, Brites D. Reduced Myelination and Increased Glia Reactivity Resulting from Severe Neonatal Hyperbilirubinemia. Mol Pharmacol. 2016 Jan;89(1):84-93.

15. Blumberg RM, Cady EB, Wigglesworth JS, McKenzie JE, Edwards AD. Relation between delayed impairment of cerebral energy metabolism and infarction following transient focal hypoxiaischaemia in the developing brain. Exp Brain Res. 1997 Jan;113(1):130-7.

16. Qi T, Wu Y, Zeng J, Zhang F, Xue A, Jiang L, Zhu Z, Kemper K, Yengo L, Zheng Z; eQTLGen Consortium, Marioni RE, Montgomery GW, Deary IJ, Wray NR, Visscher PM, McRae AF, Yang J. Identifying gene targets for brain-related traits using transcriptomic and methylomic data from blood. Nat Commun. 2018 Jun 11;9(1):2282.

17. Ashburner M, Ball CA, Blake JA, Botstein D, Butler H, Cherry JM, Davis AP, Dolinski K, Dwight SS, Eppig JT, Harris MA, Hill DP, Issel-Tarver L, Kasarskis A, Lewis S, Matese JC, Richardson JE, Ringwald M, Rubin GM, Sherlock G. Gene ontology: tool for the unification of biology. The Gene Ontology Consortium. Nat Genet. 2000 May;25(1):25-9.

18. Ogata H, Goto S, Sato K, Fujibuchi W, Bono H, Kanehisa M. KEGG: Kyoto Encyclopedia of Genes and Genomes. Nucleic Acids Res. 1999 Jan 1;27(1):29-34.

19. X Jiao, BT Sherman, R Stephens, MW Baseler, HC Lane, RA Lempicki. DAVID-WS: a stateful web service to facilitate gene/protein list analysis. Bioinformatics (2012) 28 (13): 1805-1806. 
20. Chen X, Xizeng M, Jiaju H, Yang D, Jianmin Wu, Shan Dong, Lei Kong, Ge Gao, Chuan-Yun Li, Liping Wei, KOBAS 2.0: a web server for annotation and identification of enriched pathways and diseases, Nucleic Acids Research, Volume 39, Issue suppl_2, 1 July 2011, Pages W316-W322.

21. Stelzer G, Rosen N, Plaschkes I, Zimmerman S, Twik M, Fishilevich S, Stein TI, Nudel R, Lieder I, Mazor Y, Kaplan S, Dahary D, Warshawsky D, Guan-Golan Y, Kohn A, Rappaport N, Safran M, Lancet D. The GeneCards Suite: From Gene Data Mining to Disease Genome Sequence Analyses. Curr Protoc Bioinformatics. 2016 Jun 20;54:1.30.1-1.30.33.

22. Wu C, Orozco C, Boyer J, Leglise M, Goodale J, Batalov S, Hodge CL, Haase J, Janes J, Huss JW, 3rd, Su Al. BioGPS: an extensible and customizable portal for querying and organizing gene annotation resources. Genome Biol. 2009;10:R130.

23. Keenan AB, Torre D, Lachmann A, Leong AK, Wojciechowicz M, Utti V, Jagodnik K, Kropiwnicki E, Wang Z, Ma'ayan A (2019) ChEA3: transcription factor enrichment analysis by orthogonal omics integration. Nucleic Acids Research.

24. Tokar T, Pastrello C, Rossos AEM, Abovsky M, Hauschild AC, Tsay M, Lu R, Jurisica I. mirDIP 4.1integrative database of human microRNA target predictions. Nucleic Acids Res. 2018 Jan 4;46(D1):D360-D370.

25. Albertsson AM, Bi D, Duan L, Zhang X, Leavenworth JW, Qiao L, Zhu C, Cardell S, Cantor H, Hagberg $\mathrm{H}$, Mallard $\mathrm{C}$, Wang $X$. The immune response after hypoxia-ischemia in a mouse model of preterm brain injury. J Neuroinflammation. 2014 Sep 5;11:153.

26. Wang Q, Lv H, Lu L, Ren P, Li L. Neonatal hypoxic-ischemic encephalopathy: emerging therapeutic strategies based on pathophysiologic phases of the injury. J Matern Fetal Neonatal Med. 2019 Nov;32(21):3685-3692.

27. Durafourt B. A., Moore C. S., Zammit D. A., Johnson T. A., Zaguia F., Guiot M. C., et al . (2012). Comparison of polarization properties of human adult microglia and blood-derived macrophages. Glia 60, 717-727.

28. Li B., Concepcion K., Meng X., Zhang L. (2017). Brain-immune interactions in perinatal hypoxicischemic brain injury. Prog. Neurobiol. 159, 50-68.

29. Bai J, Zeng S, Zhu J, Fu C, He M, Zhu J, Chen S, Fu X, Li P, Lin Z. The Small Molecule P7C3-A20 Exerts Neuroprotective Effects in a Hypoxic-ischemic Encephalopathy Model via Activation of PI3K/AKT/GSK3ß Signaling. Neuroscience. 2020 Aug 10;441:197-208.

30. Jeong JE, Park JH, Kim CS, Lee SL, Chung HL, Kim WT, Lee EJ. Neuroprotective effects of erythropoietin against hypoxic injury via modulation of the mitogen-activated protein kinase pathway and apoptosis. Korean J Pediatr. 2017 Jun;60(6):181-188.

31. Kanehisa, M. and Sato, Y. (2020) KEGG Mapper for inferring cellular functions from protein sequences. Protein Sci. 29, 28-35.

32. Ginsberg MH. Integrin activation. BMB Rep. 2014 Dec;47(12):655-9.

33. Hou M, Cui J, Liu J, Liu F, Jiang R, Liu K, Wang Y, Yin L, Liu W, Yu B. Angiopoietin-like 4 confers resistance to hypoxia/serum deprivation-induced apoptosis through PI3K/Akt and ERK1/2 signaling 
pathways in mesenchymal stem cells. PLoS One. 2014 Jan 21;9(1):e85808.

34. Bona E, Andersson AL, Blomgren K, Gilland E, Puka-Sundvall M, Gustafson K, Hagberg H. Chemokine and inflammatory cell response to hypoxia-ischemia in immature rats. Pediatr Res. 1999 Apr;45(4 Pt 1):500-9.

35. Fan X, Wang H, Zhang L, Tang J, Qu Y, Mu D. Neuroprotection of hypoxic/ischemic preconditioning in neonatal brain with hypoxic-ischemic injury. Rev Neurosci. 2020 Aug.

36. Bassett JD, Yang TC, Bernard D, Millar JB, Swift SL, McGray AJ, VanSeggelen H, Boudreau JE, Finn JD, Parsons R, Evelegh C, Damjanovic D, Grinshtein N, Divangahi M, Zhang L, Xing Z, Wan Y, Bramson JL. CD8+ T-cell expansion and maintenance after recombinant adenovirus immunization rely upon cooperation between hematopoietic and nonhematopoietic antigen-presenting cells. Blood. 2011;117:1146-1155.

37. Albertsson AM, Bi D, Duan L, Zhang X, Leavenworth JW, Qiao L, Zhu C, Cardell S, Cantor H, Hagberg $\mathrm{H}$, Mallard $\mathrm{C}$, Wang $\mathrm{X}$. The immune response after hypoxia-ischemia in a mouse model of preterm brain injury. J Neuroinflammation. 2014 Sep 5;11:153.

38. Wilhelmsson U, Andersson D, de Pablo Y, Pekny R, Ståhlberg A, Mulder J, Mitsios N, Hortobágyi T, Pekny M, Pekna M. Injury Leads to the Appearance of Cells with Characteristics of Both Microglia and Astrocytes in Mouse and Human Brain. Cereb cortex tissue. 2017 Jun 1;27(6):3360-3377.

39. Chu X, Cao L, Yu Z, Xin D, Li T, Ma W, Zhou X, Chen W, Liu D, Wang Z. Hydrogen-rich saline promotes microglia $\mathrm{M} 2$ polarization and complement-mediated synapse loss to restore behavioral deficits following hypoxia-ischemic in neonatal mice via AMPK activation. J Neuroinflammation. 2019 May 18;16(1):104.

40. Kinugawa K, Monnet Y, Béchade C, Alvarez-Fischer D, Hirsch EC, Bessis A, Hunot S. DAP12 and $\mathrm{CD} 11 \mathrm{~b}$ contribute to the microglial-induced death of dopaminergic neurons in vitro but not in vivo in the MPTP mouse model of Parkinson's disease. J Neuroinflammation. 2013 Jul 11;10:82.

41. Kuan CY, Chen HR, Gao N, Kuo YM, Chen CW, Yang D, Kinkaid MM, Hu E, Sun YY. Brain-targeted hypoxia-inducible factor stabilization reduces neonatal hypoxic-ischemic brain injury. Neurobiol Dis. 2021 Jan;148:105200.

42. Li H, Liu B, Gu C, Zeng X, Liu Y, Zhang S, Gong H, Shao Y, Yao Z, An R. Relations of neuropeptide Y and heme oxygenase- 1 expressions with fetal brain injury in rats with intrahepatic cholestasis of pregnancy. Acta Cir Bras. 2019 May 6;34(4):e201900401.

43. Yang L, Jiang Y, Wen Z, Xu X, Xu X, Zhu J, Xie X, Xu L, Xie Y, Liu X, Xu G. Over-expressed EGR1 may exaggerate ischemic injury after experimental stroke by decreasing BDNF expression. Neuroscience. 2015 Apr 2;290:509-17.

44. Catalanotto C, Cogoni C, Zardo G. MicroRNA in Control of Gene Expression: An Overview of Nuclear Functions. Int J Mol Sci. 2016 Oct 13;17(10):1712.

45. Looney AM, Walsh BH, Moloney G, Grenham S, Fagan A, O'Keeffe GW, Clarke G, Cryan JF, Dinan TG, Boylan GB, Murray DM. Downregulation of Umbilical Cord Blood Levels of miR-374a in Neonatal Hypoxic Ischemic Encephalopathy. J Pediatr. 2015 Aug;167(2):269-73.e2. 
46. Yang $X$, Li D, Qi YZ, Chen W, Yang CH, Jiang YH. MicroRNA-217 ameliorates inflammatory damage of endothelial cells induced by oxidized LDL by targeting EGR1. Mol Cell Biochem. 2020 Dec;475(12):41-51.

47. Yao Y, Fan X, Yu B, Li T, Zhang Y. Knockdown of long noncoding RNA Malat1 aggravates hypoxiainduced cardiomyocyte injury by targeting miR-217. Adv Clin Exp Med. 2019 Jun;28(6):719-728.

\section{Tables}

Table 1 Information on the datasets included in the current study.

\begin{tabular}{|lllll|}
\hline Dateset & Platform & No. of samples(normal/HIBD) & Tissue & Species \\
\hline GSE37777 & GPL7294 & $3 / 3$ & cerebral cortical & rats \\
\hline GSE18356 & GPL7294 & $4 / 4$ & cerebral cortical & rats \\
\hline GSE121178 & GPL21220 & $3 / 3$ & whole blood & human \\
GSE112137 & GPL20301 & $16 / 16$ & cortical progenitors cells & human \\
\hline
\end{tabular}

Table 2. The Gene Ontology (GO) functions and Kyoto Encyclopedia of Genes and Genomes (KEGG) pathways enriched for the genes involved in 2 modules .

\begin{tabular}{|llll|}
\hline Description & P Value & Count & Gene symbol \\
\hline G0:0007229 integrin-mediated signaling pathway & $5.08 \mathrm{E}-04$ & 3 & $\begin{array}{l}\text { FGR, ITGAM, } \\
\text { TYROBP }\end{array}$ \\
\hline $\begin{array}{l}\text { G0:0045080 positive regulation of chemokine } \\
\text { biosynthetic process }\end{array}$ & 0.003568345 & 2 & EGR1,HMOX1 \\
\hline $\begin{array}{l}\text { G0:0001666 response to hypoxia } \\
\text { G0:0050776 regulation of immune response }\end{array}$ & 0.059914081 & 2 & EGR1,HMOX1 \\
\hline $\begin{array}{l}\text { hsa04066 HIF-1 signaling pathway } \\
\text { hsa05225 Hepatocellular carcinoma }\end{array}$ & 0.000509333 & 2 & HMOX1,HK3 \\
\hline hsa04015 Rap1 signaling pathway & 0.001153006 & 2 & HMOX1,HGF \\
\hline $\begin{array}{l}\text { hsa00524 Neomycin, kanamycin and gentamicin } \\
\text { biosynthesis }\end{array}$ & 0.001821363 & 2 & ITGAM,HGF \\
\hline hsa00052 Galactose metabolism & 0.001850459 & 1 & HK3 \\
\hline
\end{tabular}


Table 3. hematologic/immune system tissue-specific potential biomarker genes in HIBD

\begin{tabular}{|llll|}
\hline Gene & Description & $\begin{array}{l}\text { Genecards } \\
\text { datebase }\end{array}$ & Biogps datebase \\
\hline TYROBP & $\begin{array}{l}\text { transmembrane immune signaling } \\
\text { adaptor }\end{array}$ & Related to HIBD & $\begin{array}{l}\text { hematologic/immune } \\
\text { system }\end{array}$ \\
\hline ITGAM & integrin subunit alpha M & Related to HIBD & $\begin{array}{l}\text { hematologic/immune } \\
\text { system }\end{array}$ \\
\hline EGR1 & early growth response 1 & Related to HIBD & $\begin{array}{l}\text { hematologic/immune } \\
\text { system }\end{array}$ \\
\hline HMOX1 & heme oxygenase 1 & Related to HIBD & $\begin{array}{l}\text { hematologic/immune } \\
\text { system }\end{array}$ \\
\hline
\end{tabular}

\section{Figures}


A

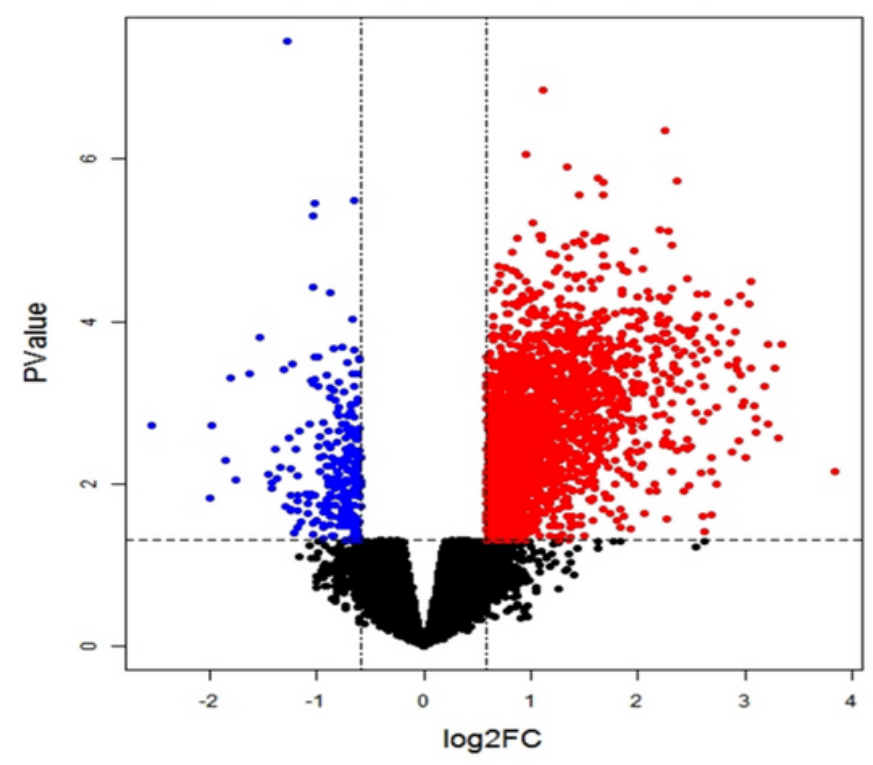

C

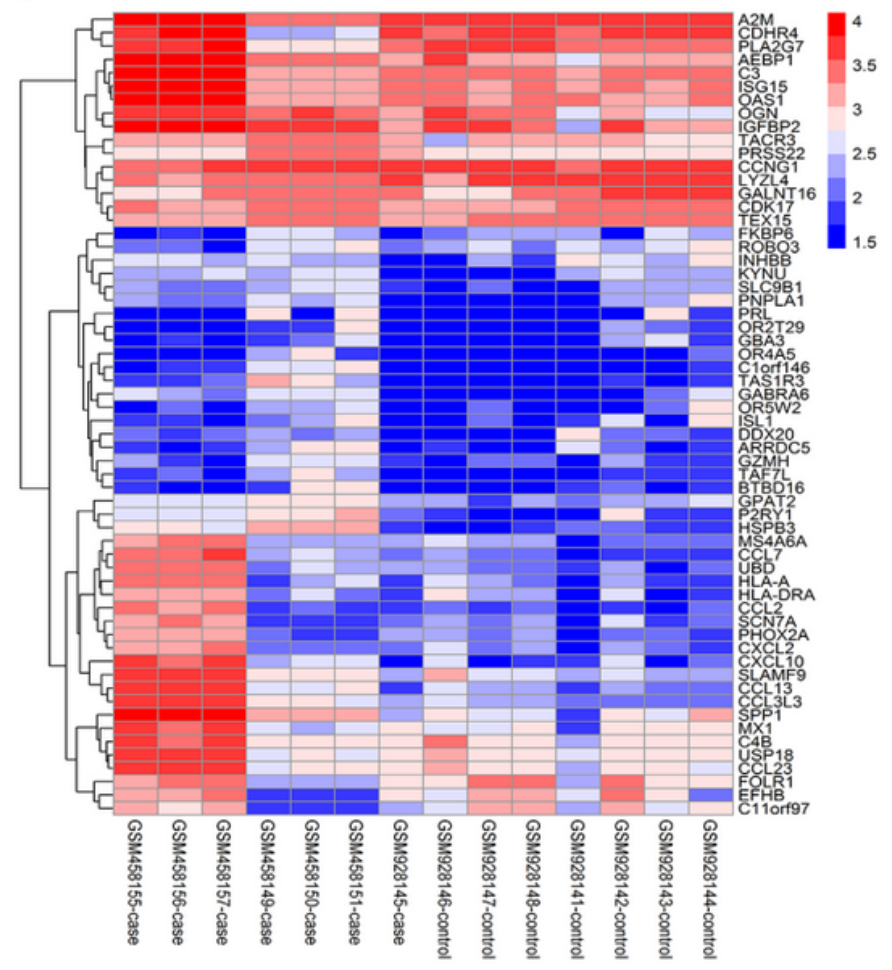

B

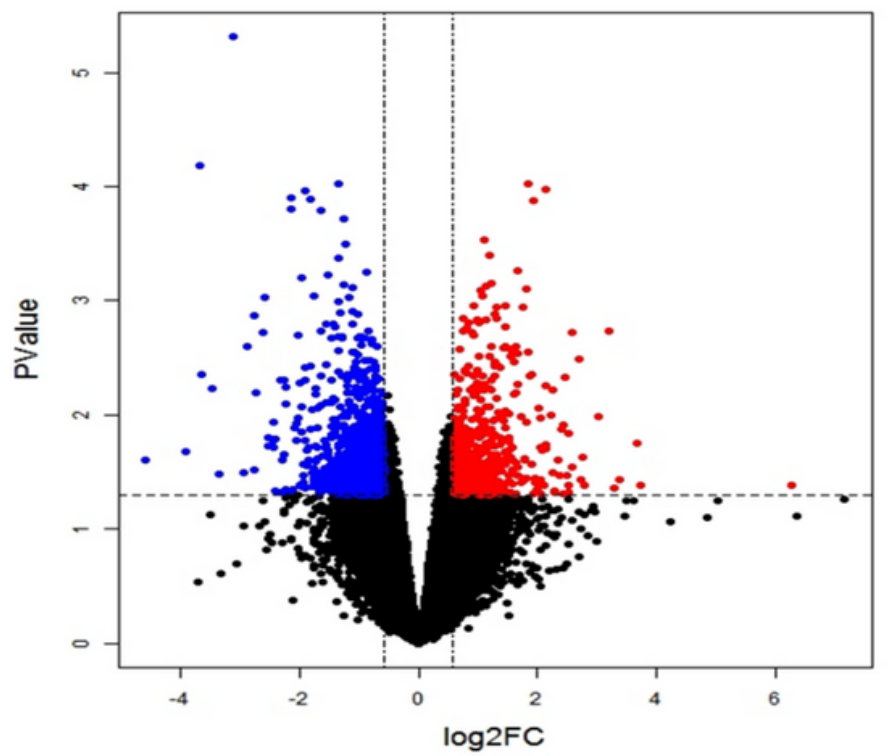

D

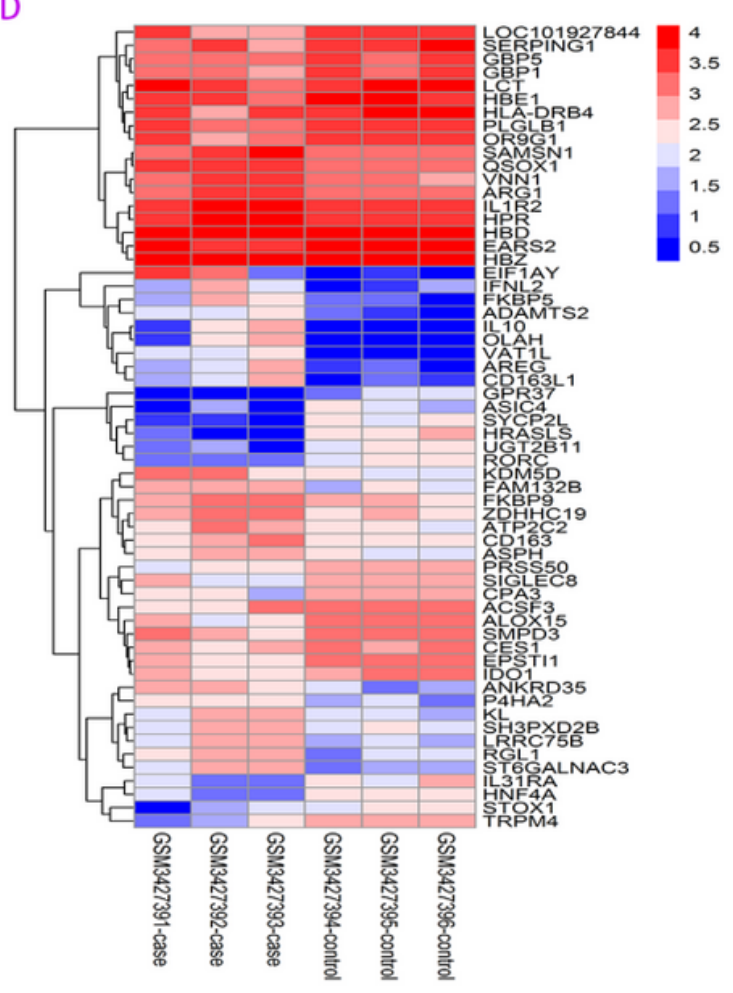

\section{Figure 1}

Differential expression analysis of mRNA of cerebral cortex and blood in HIBD.(A,B)Volcano map of DEGs in the cerebral cortex tissue and blood. (C,D) Heatmap of the top 30 up-regulated DEGs and top 30 downregulated DEGs in the cerebral cortex tissue and blood. Red represents up-regulated genes and blue represents down-regulated genes. 


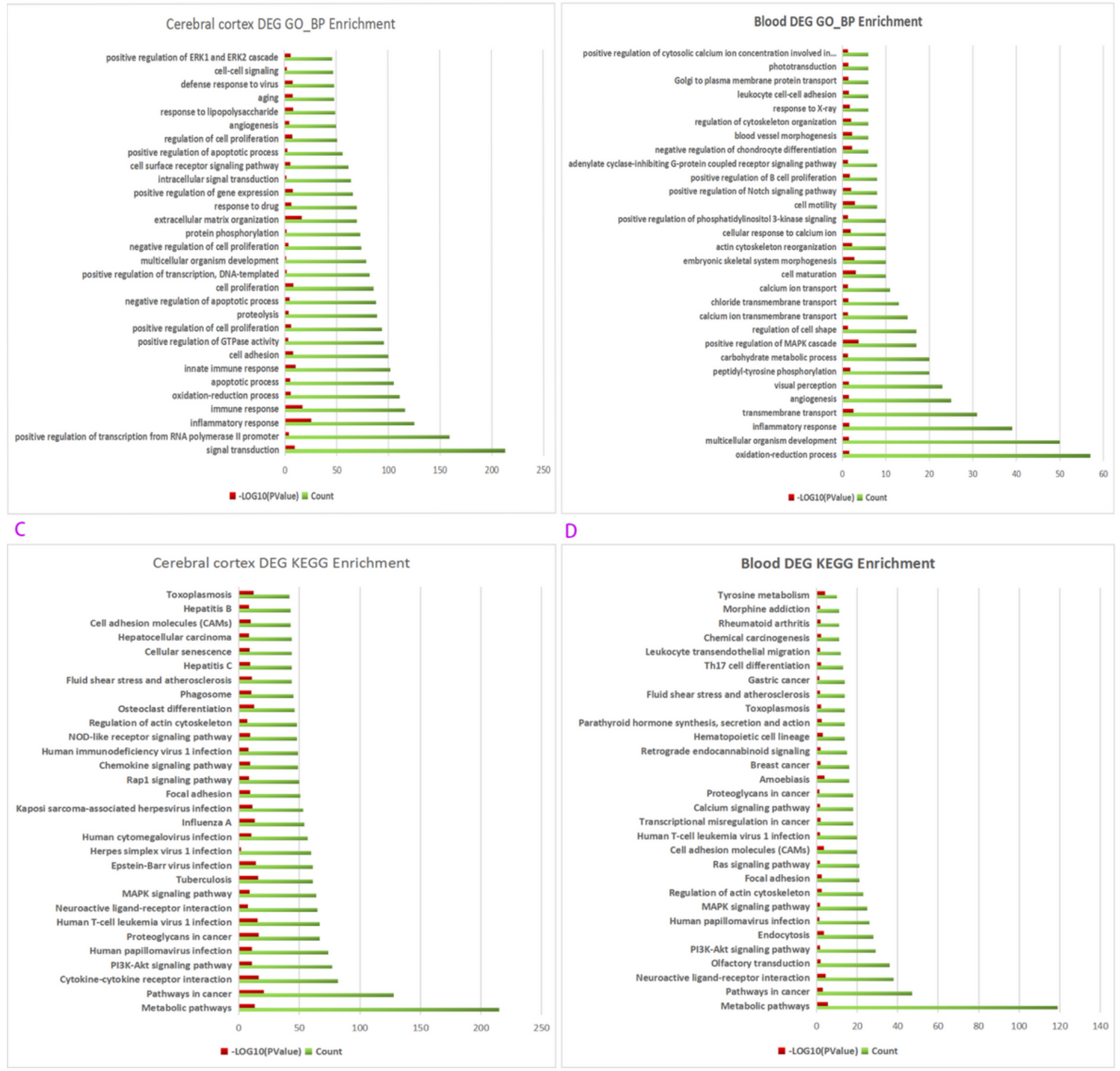

\section{Figure 2}

Enrichment analysis. (A,B)GO annotation of DEGs in the cerebral cortex tissue and blood. (C,D)KEGG pathway enrichment analysis of DEGs in the cerebral cortex tissue and blood. The results of enrichment analysis of DEGs in the two kinds of samples were both involved in inflammatory response, immune response and apoptosis. 


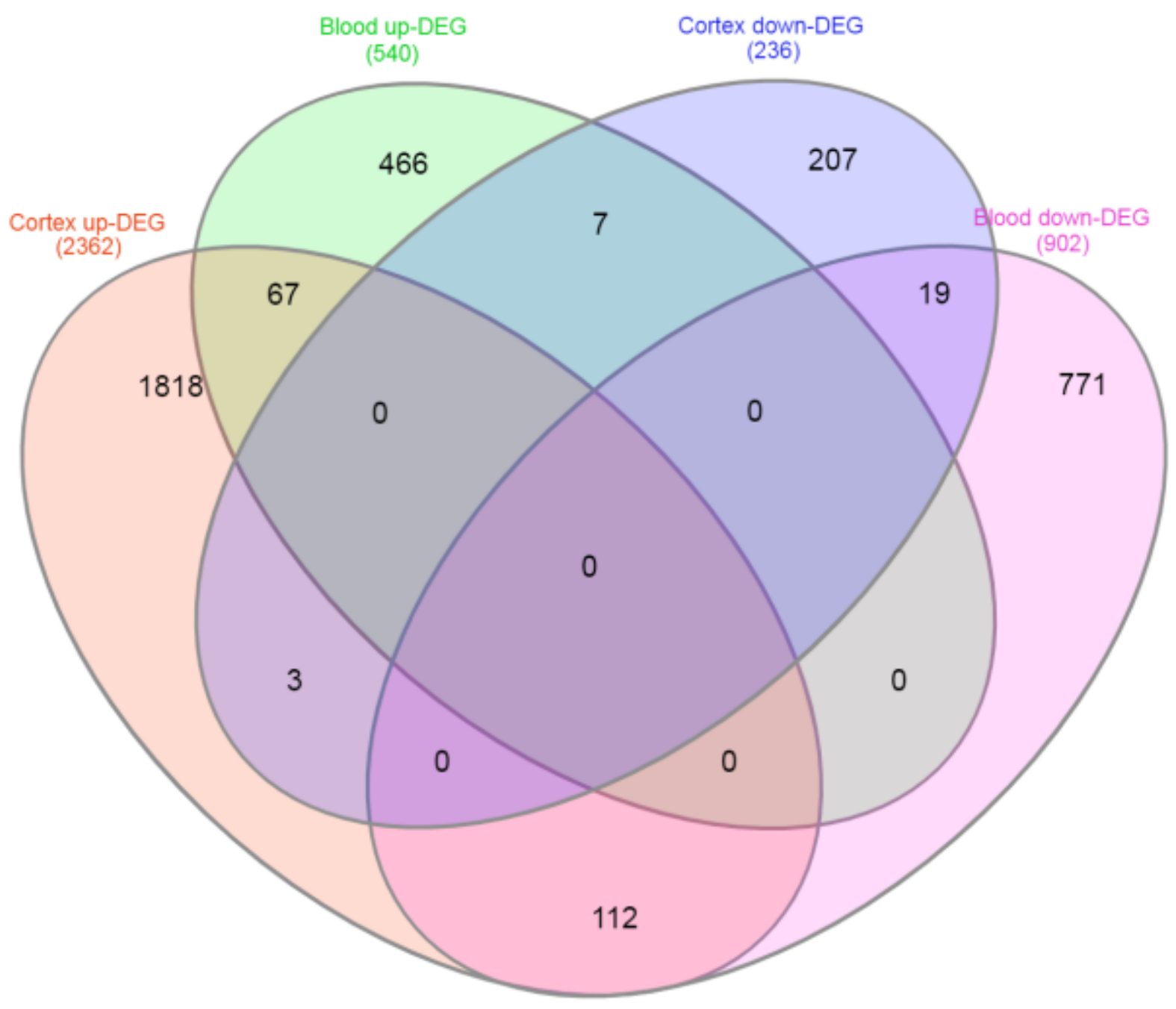

Figure 3

Venn diagram of the DEGs in the cerebral cortex tissue and blood. A total of 86 common genes were obtained. 


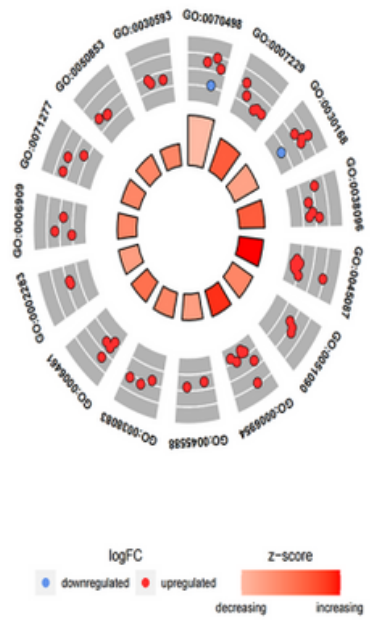

C

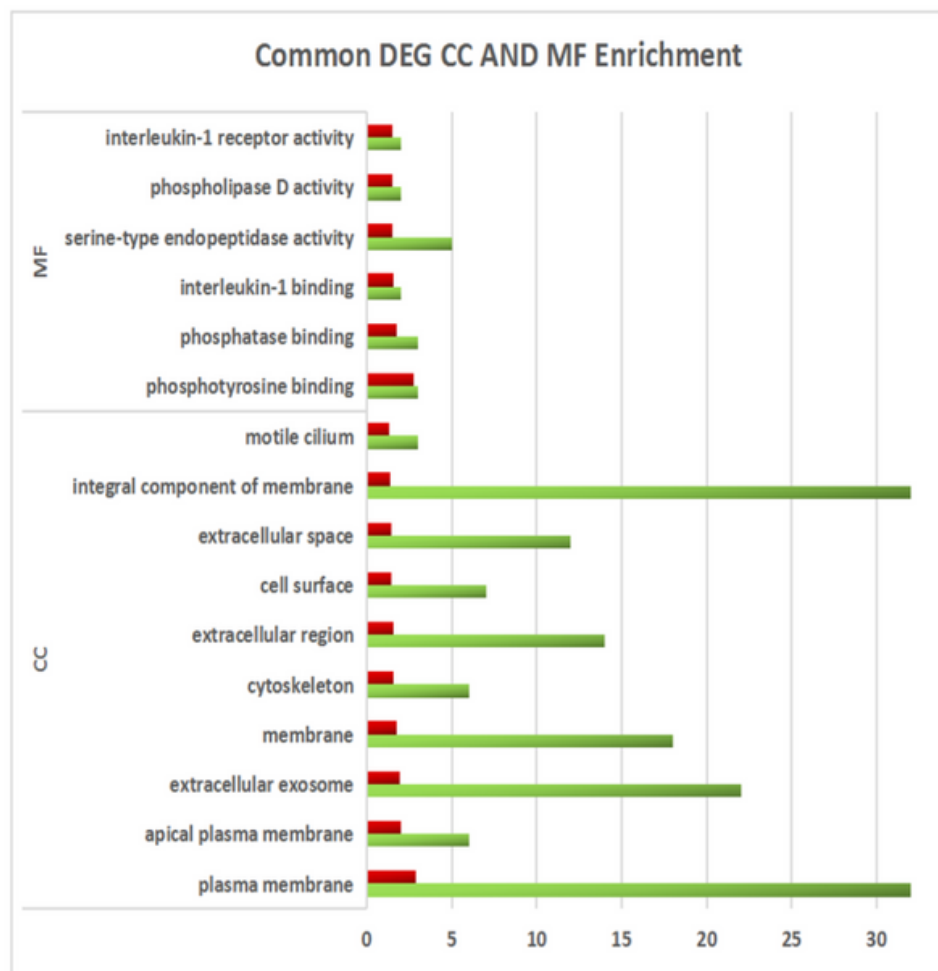

\begin{tabular}{|c|c|}
\hline 10 & Description \\
\hline G0:0070498 & interleukin-1-mediated signaling pattwway \\
\hline G0:0007229 & integrin-mediated signaling pathway \\
\hline GO:0030168 & platelet activation \\
\hline 60:0038096 & Fc-gamma receptor signaling pattway involved in phagocylosis \\
\hline 60:0045087 & innate immune response \\
\hline 60:0051090 & regulation of sequence-specific ONA binding transcipition factor accivinty \\
\hline $60: 0006954$ & infammatory response \\
\hline G0:0045588 & positive regulasion of gamma-delta $T$ cell differentiation \\
\hline 60:0038083 & peptioyl-yrosine autophosphorylation \\
\hline 60:0006461 & protein complex assemby \\
\hline 60:0002283 & neutrophil activation imolved in immune response \\
\hline $60: 0006009$ & phagocylosis \\
\hline 60:0071277 & celluar response to calcum ion \\
\hline 60:0050853 & B cell receptor signaling pattway \\
\hline G0:0030593 & neutrophil chemolaxis \\
\hline
\end{tabular}
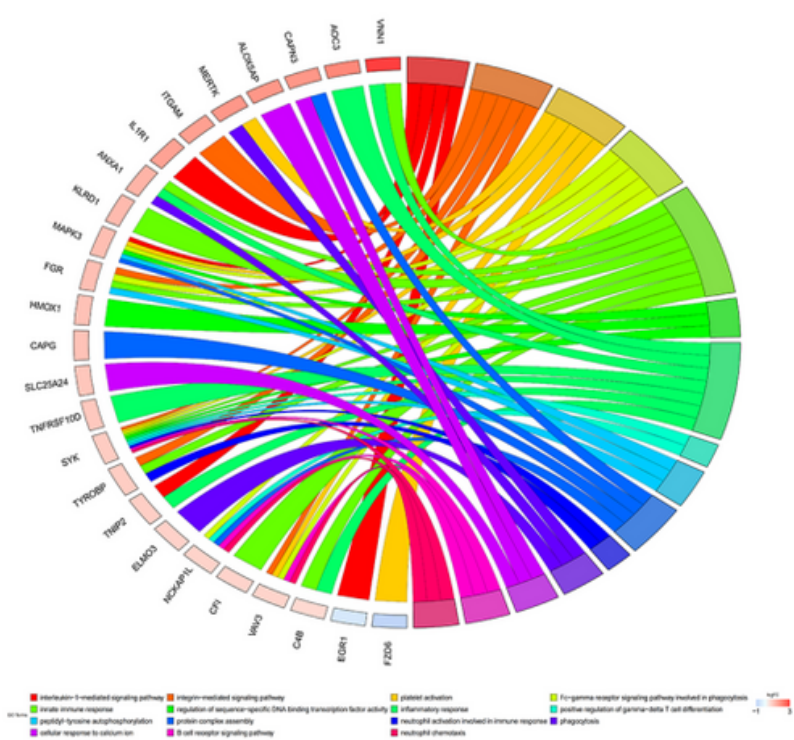

D

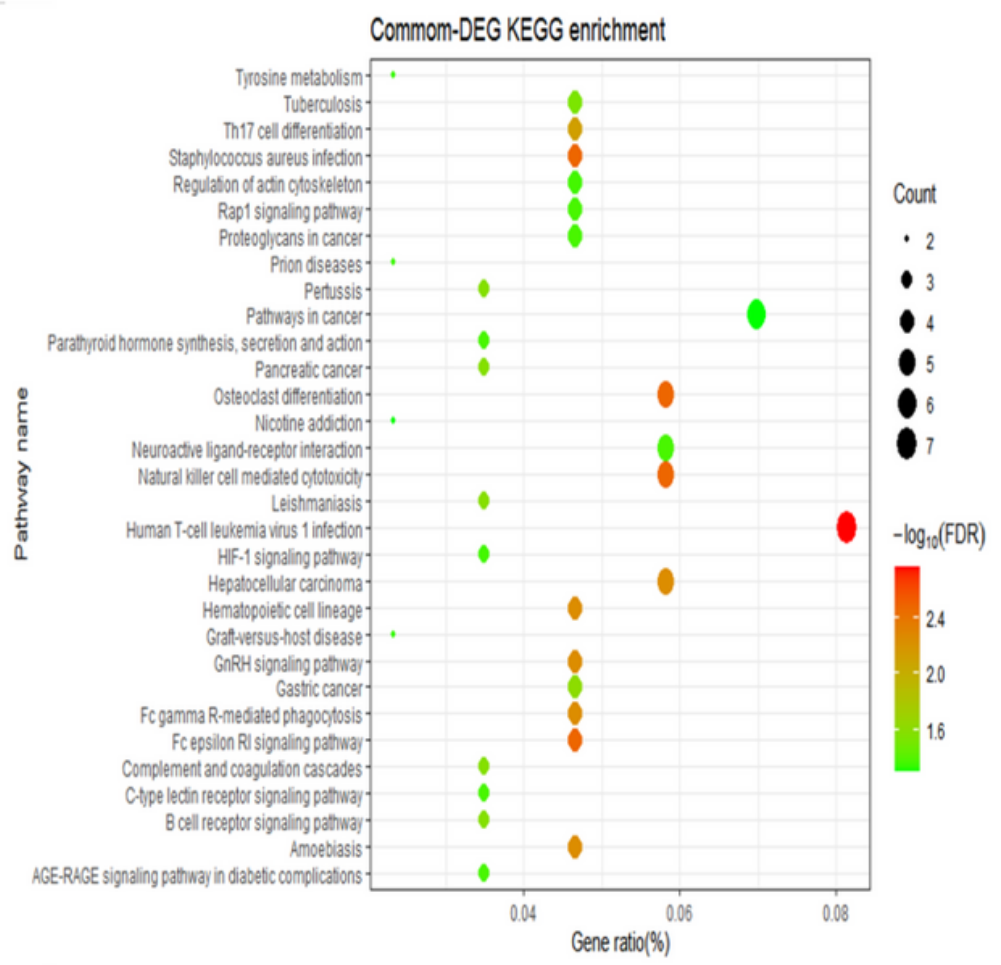

\section{Figure 4}

Enrichment analysis of common DEGs. $(A, B, C) G O$ annotation of the Common DEGs . Biological process mainly included participation in neutrophils, macrophage-mediated inflammation, platelet activation, inflammatory cells activation,participates in immune response, regulation of lymphocyte differentiation; cellular components mainly included plasma membrane, extracellular body, cytoskeleton, extracellular zone, cell surface, extracellular space, and oscillating cilia; molecular functions mainly included binding to phosphotyrosine, phosphatase, interleukin-1, serine endopeptidase activity, phospholipase $D$ activity, 
interleukin-1 receptor activity. (D)KEGG pathways mostly included microbial infection, natural killer cellmediated cytotoxicity, osteoclast differentiation, cancer pathway, FcERI signaling pathways, complement and coagulation cascade pathways, hematopoietic cell spectrum, neuroactive ligand receptor interaction, Rap1 signaling pathway.

A

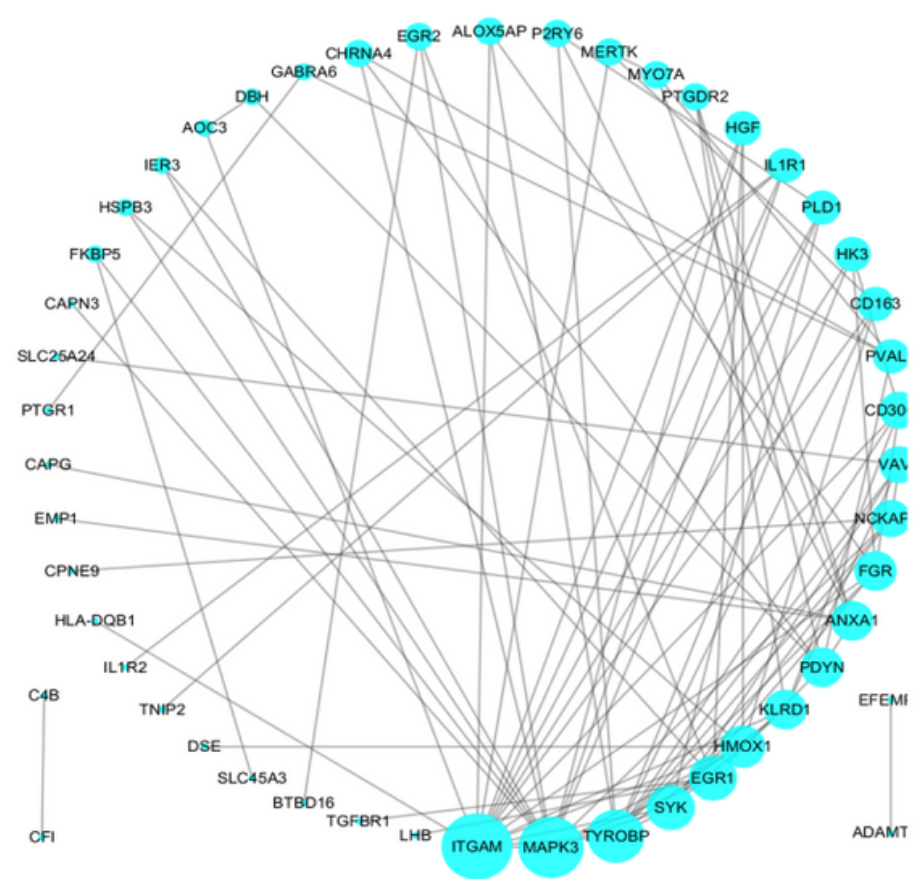

C

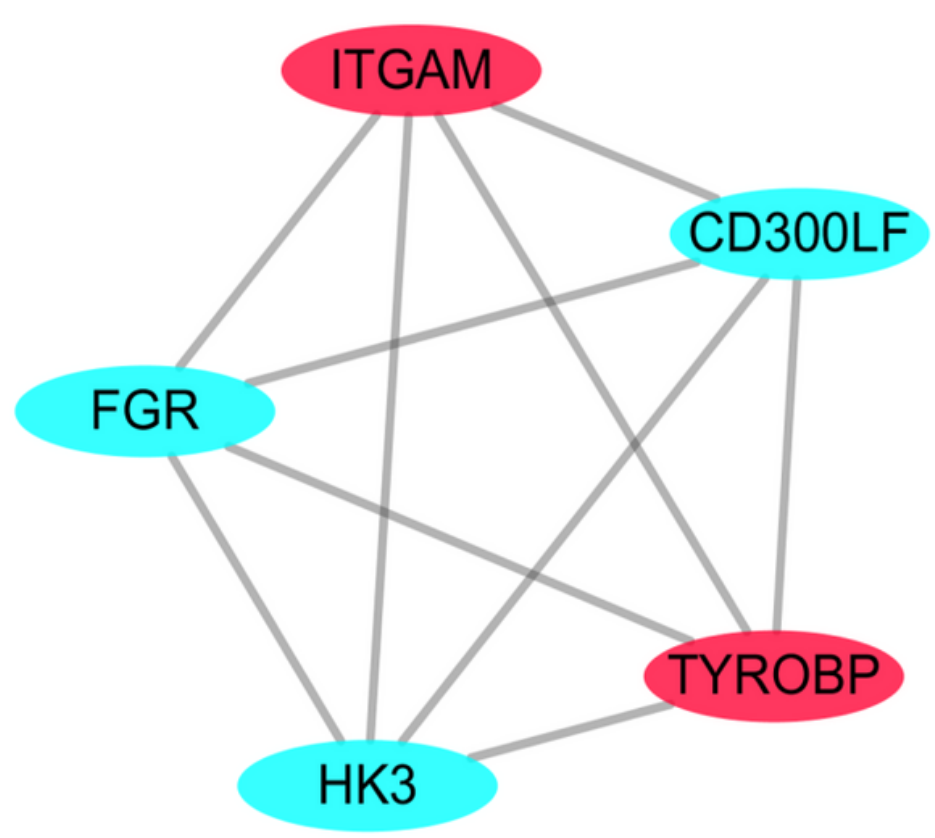

B

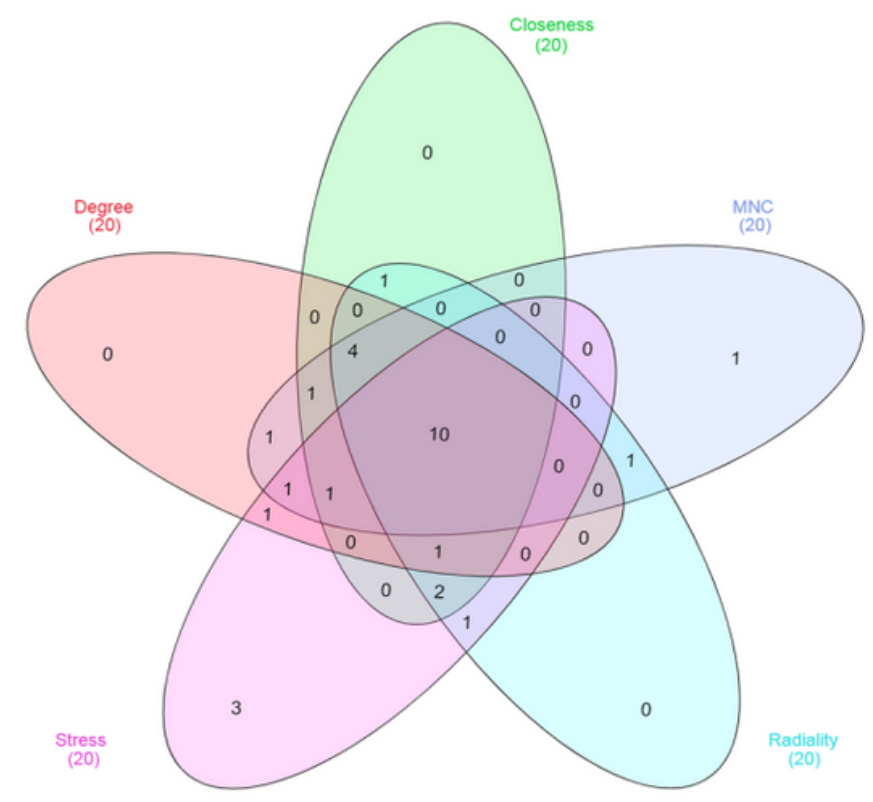

D

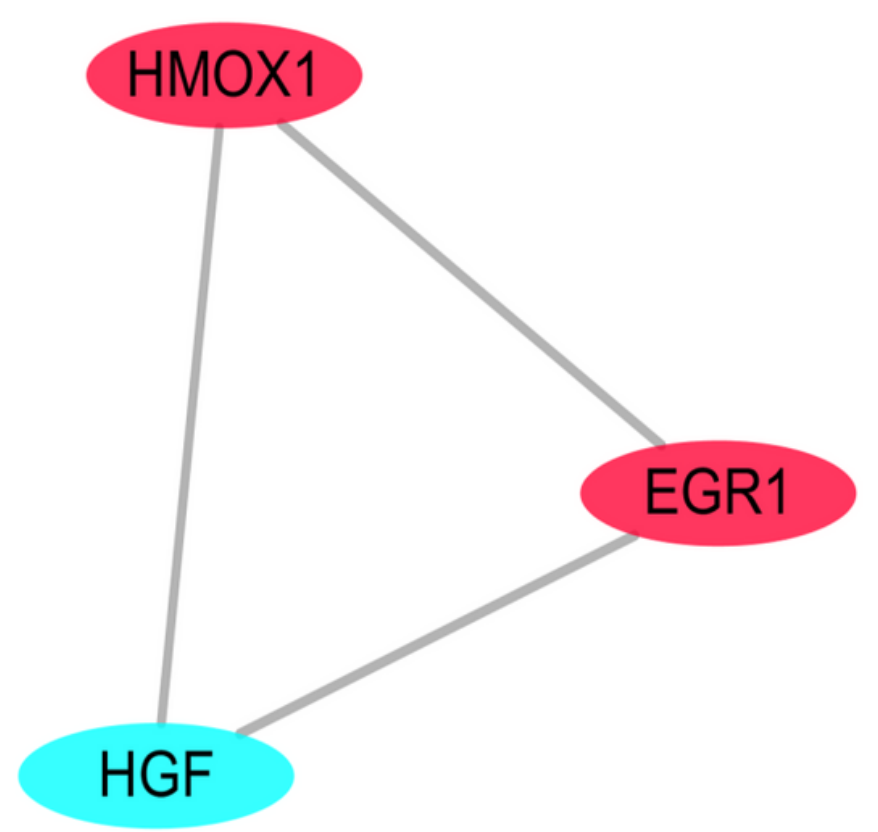

Figure 5

The results of protein-protein interaction network. (A)The PPI network of 86 common DEGs. The larger the Degree, the larger the dot. (B)Venn diagram of the result of screening central genes by five centrality 
methods methods(degree, Maximum Neighborhood Component (MNC), Radiality centrality, Stress centrality, Closeness centrality).(C,D)Two key modules were selected from the PPI network(Cluster1=3.70, Cluster2=2.80); It contains 8 common DEGs.

A

\section{EGR1}

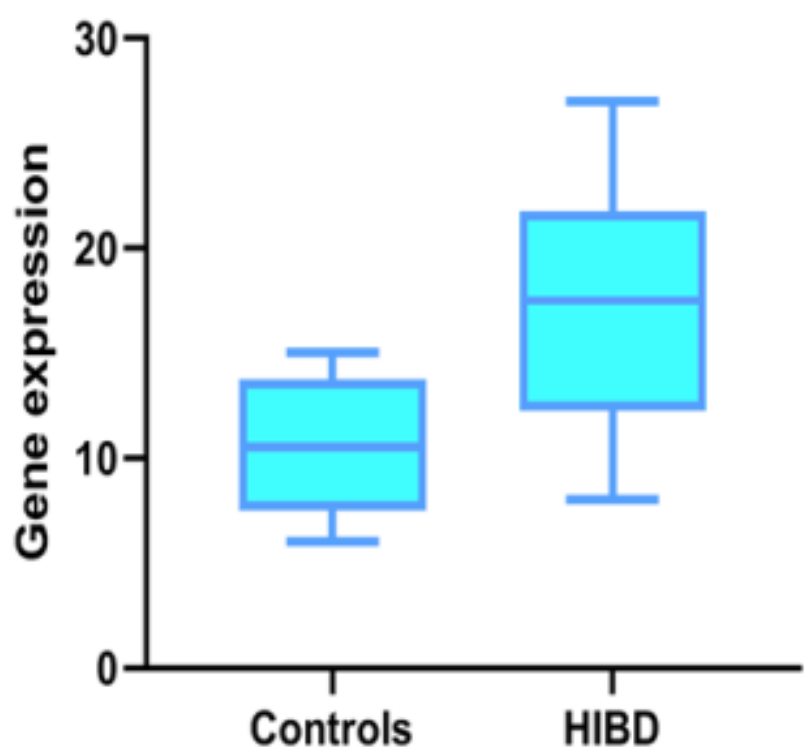

C
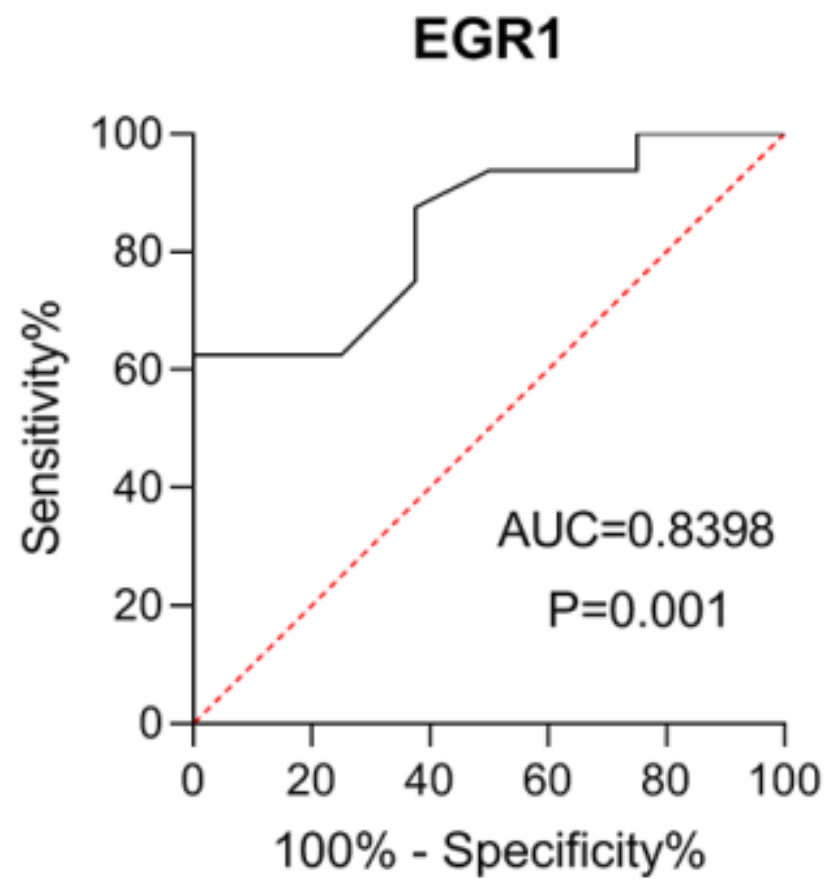

B

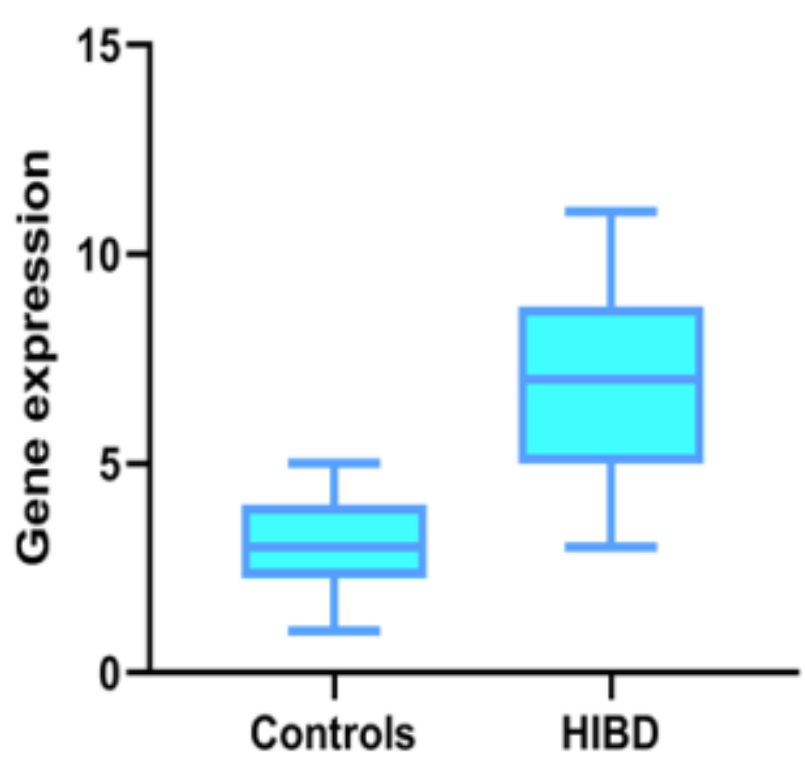

D

HMOX1

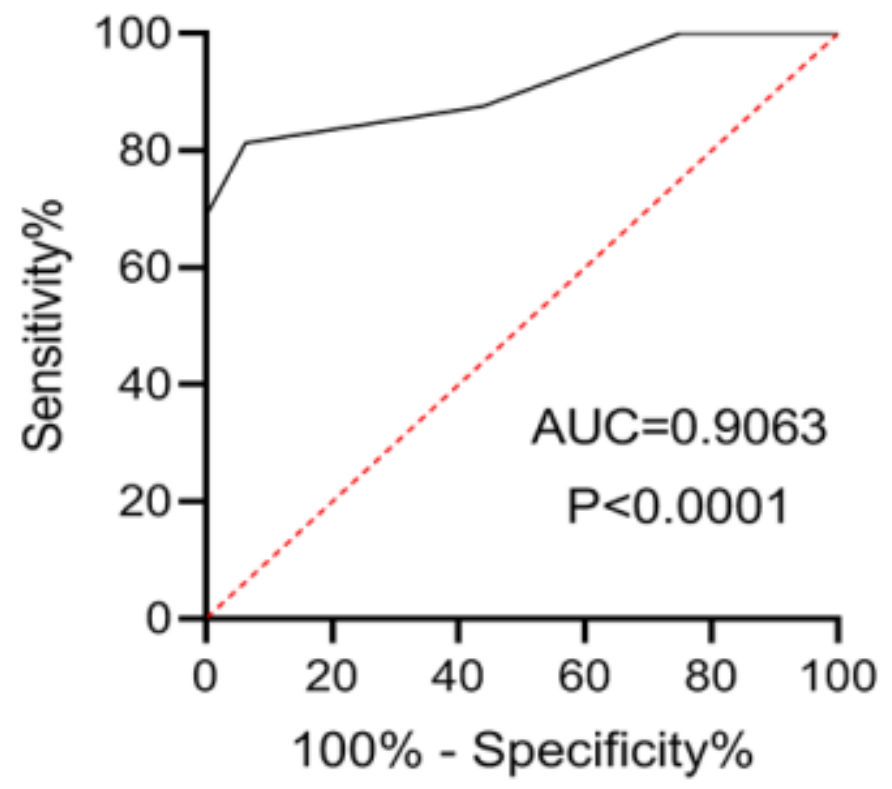

Figure 6

External verification of tissue-specific genes. $(A, B) B o x-p l o t$ of EGR1 and HMOX1. Both key central genes were significantly up-regulated in the validation data set.(C,D)Predicted ROC curves of EGR1 and HMOX1.Both key central genes have good performance in identifying cases and controls \AUCEGR1 $=0.8398, A U C H M O X 1=0.9063)$. 
A

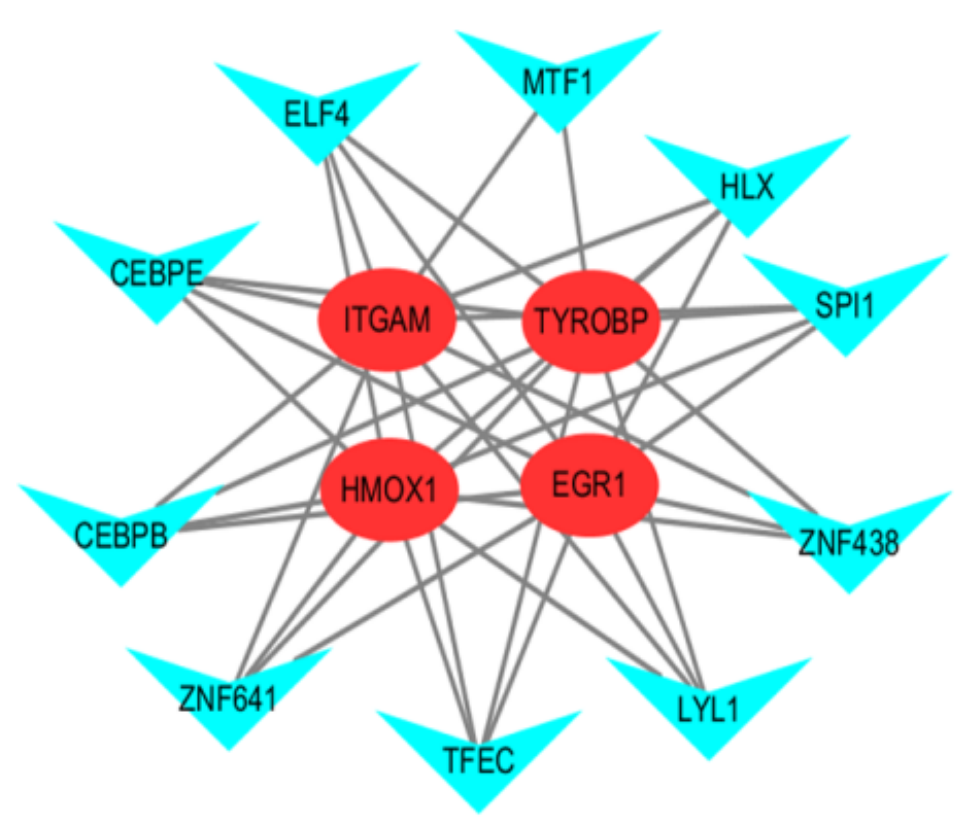

B

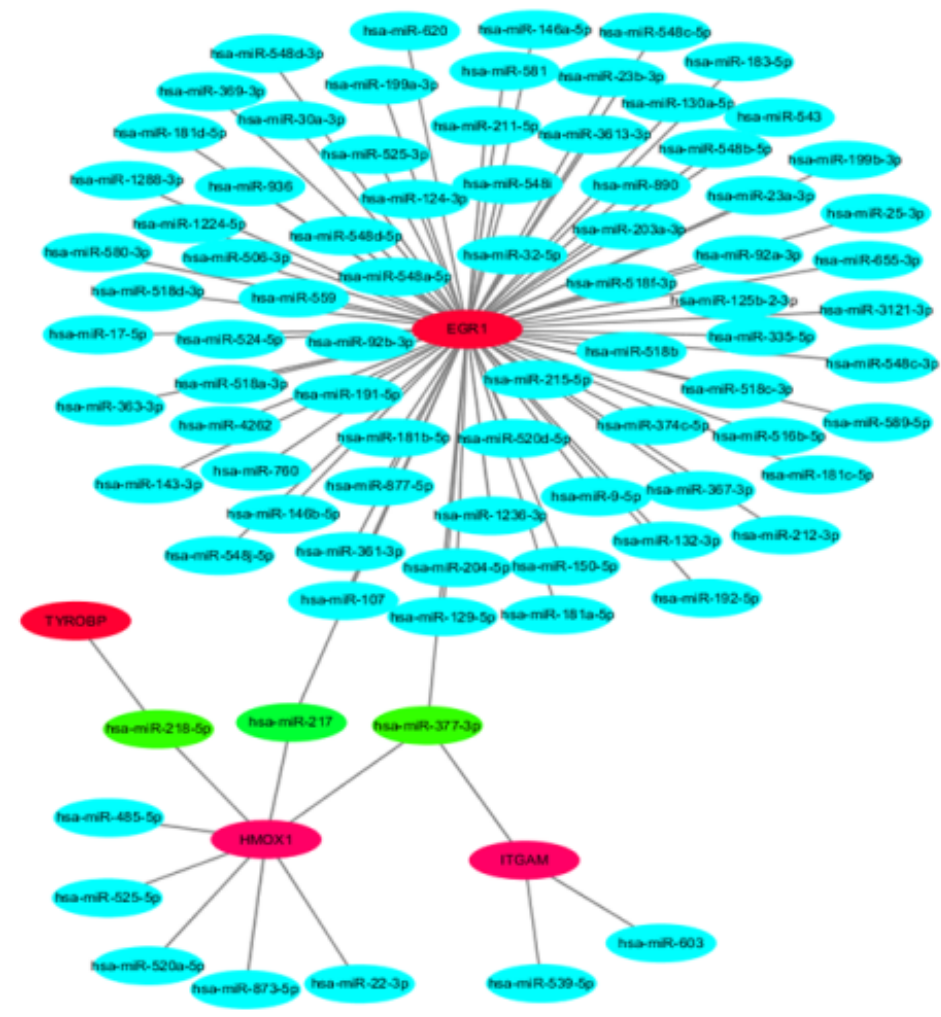

\section{Figure 7}

The interaction network of key central gene-transcription factor and key central gene-miRNA.(A)9 key transcription factors (TFEC, SPI1, CEBPE, HLX, ZNF438, ZNF641, CEBPB, ELF4, LYL1)were identified. (B)3 key miRNAs (hsa-miR-218-5p, hsa-miR-217, hsa-miR-377-3p) were identified

\section{Supplementary Files}

This is a list of supplementary files associated with this preprint. Click to download.

- Supplementary.zip 